\title{
A Computer Diagnosing System of Dementia Using Smooth Pursuit Oculogyration
}

\author{
Ichiro Fukumoto \\ Institute of Biomedical Engineering, Nagaoka University of Technology \\ Kamitomioka 1603, Nagaoka, Niigata, Japan \\ ichiro@vos.nagaokaut.ac.jp \\ http://bio.nagaokaut.ac.jp/ $\sim_{\text {fukumoto/ }}$
}

\begin{abstract}
Human smooth pursuit eye movement has been studied aiming to develop a new dementia diagnosing system. The glass-type measuring unit was installed with a small LCD monitor and a CCD digital camera as well as a built-in image-processing unit. The subjects are 19 demented patients, 18 normal elders and 7 healthy young volunteers. The adopted velocities of pursuing target are 300, 400 and 500 pixel/s. As diagnosing parameters, we have adopted the switching time of internal \& external rectus muscles, max velocities and peaks of oculogyration. As a conclusion, the real-time measurement of human oculogyration seems to become a new diagnosing method of the dementia because the smooth pursuit of the demented patients has presented clear deviations from the healthy elders. Especially the pursuit velocity, peak value, switching time and correlation coefficient seems to be very useful to discriminate the demented patients from the healthy elder subjects objectively.
\end{abstract}

\section{Introduction}

Human beings use eye movement (oculogyration) not only to change his surrounding view but also to construct the information base of his conduct. It is widely admitted that aging may degrade the accommodating ability of internal eye muscles and the driving system for the external eye muscles' mobility. In 1980-1984 the abnormality of patients' oculogyration with Alzheimer type dementia were successively found out, that had opened a new panorama of dementia research based on physiological phenomena. 12 There are two different types of oculogyration, namely saccade eye movement and smooth pursuit eye movement. Most of the traditional researches are mainly concerned to the former, but the results of many researchers are not consistent. 345 On the other hand, the latter is rather difficult in measuring as well as extracting useful parameters, partly because of the wave complexity overlapping with inevitable overwhelming saccades and partly because of its physiological ambiguity. We have since 1996 studied the dementia diagnosing system using the displacements of accommodative ability such as light reflexes. 6 6 7 8 ] The developed system was installed as a prototype set and it could get the preliminary results with discriminating accuracy of $90.5 \%$. In order to improve the system further, we have tried to apply the

T. Dohi and R. Kikinis (Eds.): MICCAI 2002, LNCS 2488, pp. 674681 2002.

(C) Springer-Verlag Berlin Heidelberg 2002 


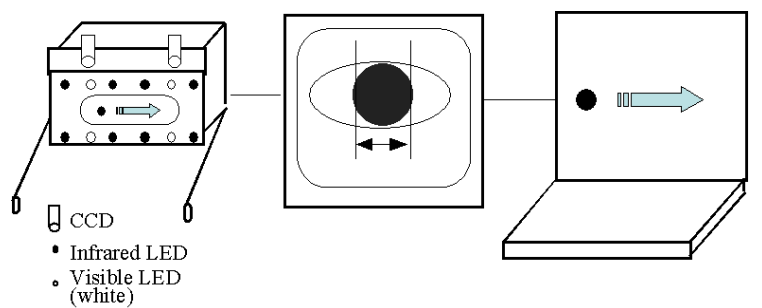

Eye glasses type Image processor Computer experimental device

Fig. 1. A block diagram of the measuring system for eye movement.

oculogyration phenomena for the compensational physiological data to evaluate the grade of dementia with further reliability. As diagnosing parameters in this study, we have adopted the switching time of internal \& external rectus muscles, max velocities and peaks of oculogyration during pursuing a target presented in a small LCD display.

\section{The Subjects and the Method}

The subjects are 19 demented patients; AD (75.1 \pm 5.9 years old, HDS-R $=11.3 \pm$ 7.7), 18 normal elders; EC (79.5 \pm 7.4 years old, HDS-R $=27.9 \pm 3.4)$ and 7 healthy young volunteers; YC $(24.9 \pm 4.8$ years old, HDS-R=30.0). Subjects are instructed to wear a glass-type attachment that includes a small LCD monitor and a CCD digital camera. They are also instructed to track a small black dot running on a horizontal line in the LCD monitor from the right to left and vice versa randomly. The velocities of pursuing target are 300, 400 and $500 \mathrm{pixel} / \mathrm{s}$. The location of an eye's pupil center is automatically calculated using cornea light reflex method by a built-in processing unit in the system through the CCD camera. Each five measured data of three types of the subject are adjusted by initial values and are rendered to visible curves by summation-averaging method. The adopted summation-averaging technique could successfully eliminate randomly inserting saccade that used to be annoying noises for the detection of the smooth pursuit eye movement. The oculogyration responses are calculated from the pupil center data afterward and are visualized as simple time versus eye-location curves in an off line batch process of connected another desktop type personal computer. As diagnosing parameters, we have adopted the switching time of internal and external rectus muscles, velocities, peak values, the averaged difference between the patient and the normal.

\section{Results and Discussions}

The measuring system is composed of three parts. (Fig.1) The first part is a glass type visual stimulating \& observing system including a LCD display and 


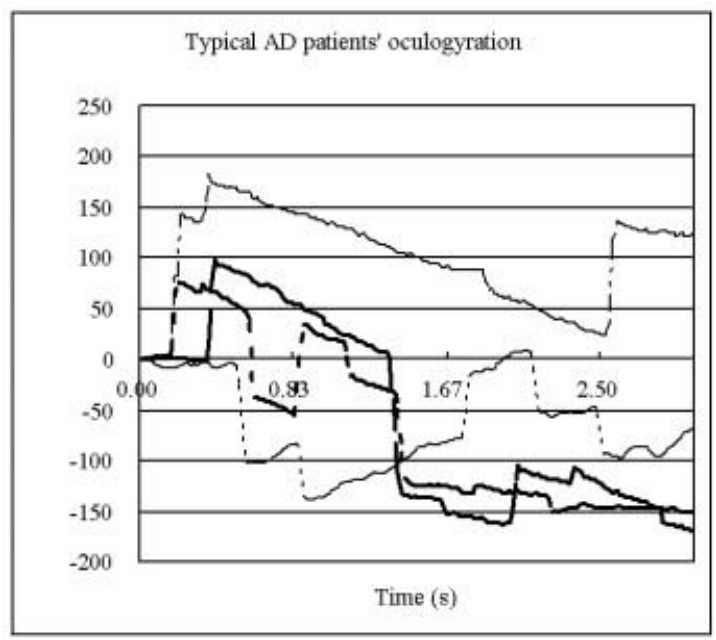

Fig. 2. Some examples of unprocessed raw data of demented patients' eye movements.

a CCD camera. Each subject is asked to wear the glass-type attachment during the measurement. A small black dot as a pursuing target runs randomly on a horizontal line in the small LCD display. The CCD camera detects the eye movement and records it time-sequentially. The second part of the system is a image-processor, which can recognize the outline of pupil from the eye image and automatically find its center. The processor calculates the eye movement that is defined as the deviation of the pupil center from the initial location during target pursuing. As is shown in Fig.2, original curves of the eye movements usually include not only smooth pursuit but also saccades. Therefore, their raw data should be processed before visualization of the desirable smooth pursuit eye movement. (Fig.2) The finally processed data of the three kinds of subjects are visualized in three graphs of different target velocities. (Fig.3, Fig.4 and Fig.5) The graphs show that the averaging is successful and that all the saccades are almost eliminated out, comparing Fig.2. From the observation of the eye movement curves, the typical patterns of smooth pursuit model of the demented, the healthy elders and the healthy young subjects are reconstructed as a simple template. (Fig.6) As far as our measured data, though we could not find any meaningful differences in delay time among the three subjects groups, we have noticed that "the switching time" shows a clear negative correlation with the delay time. (Fig.7) We have here defined the peak time as "the switching time" of two rectus eye muscles. Additionally we have found the following remarks by observing the Fig.3, Fig.4 and Fig.5.

1. The tracking velocity has a tendency that the AD patients' tracking speed is slower than the normal elders', which is further slower than the normal young subjects'. (Fig.8) 


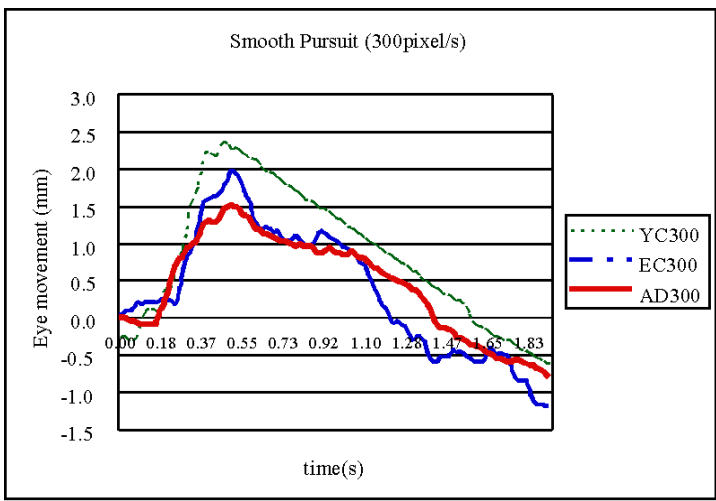

Fig. 3. The averaged smooth pursuit in 300 pixels/s target velocity.

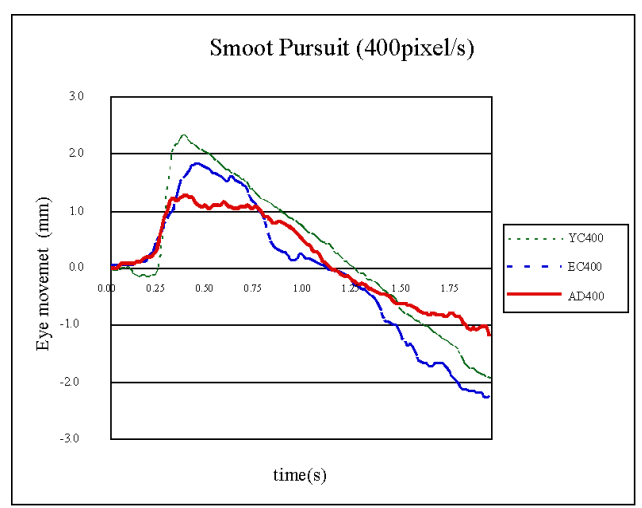

Fig. 4. The averaged smooth pursuit in 400 pixels/s target velocity.

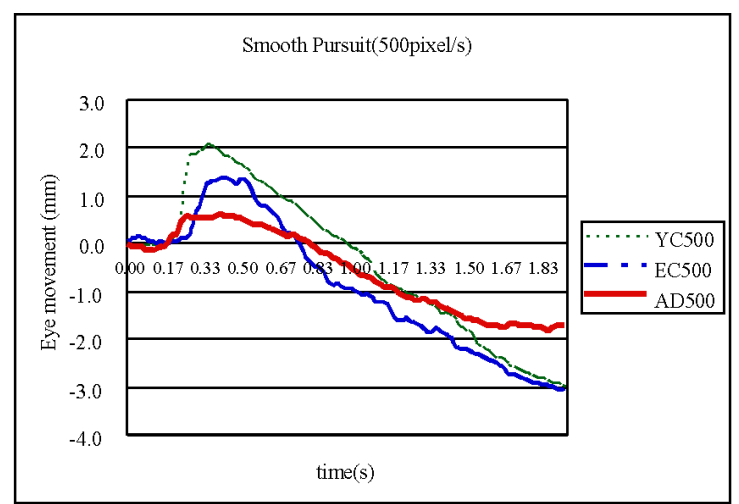

Fig. 5. The averaged smooth pursuit in 500 pixels/s target velocity. 


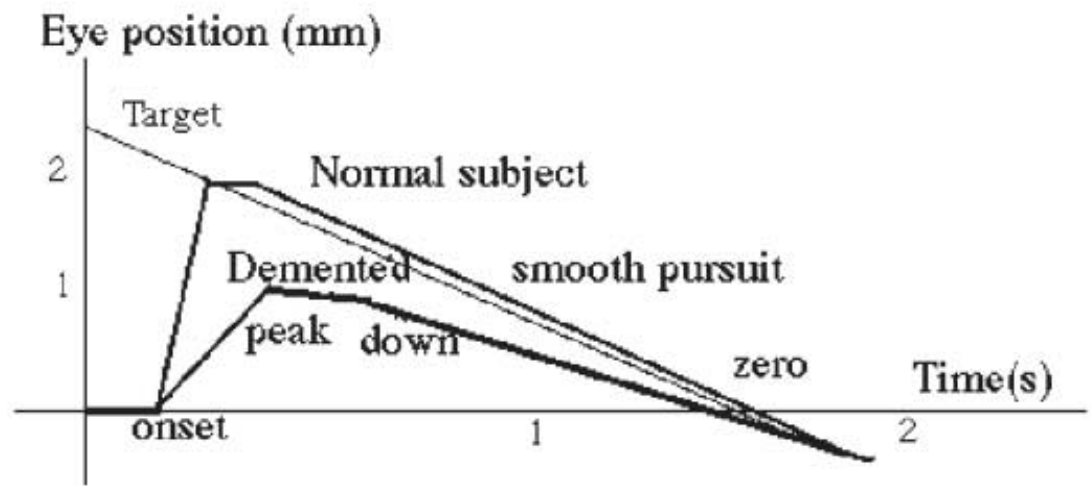

Fig. 6. A simplified pattern of smooth pursuit model from AD, EC and YC.

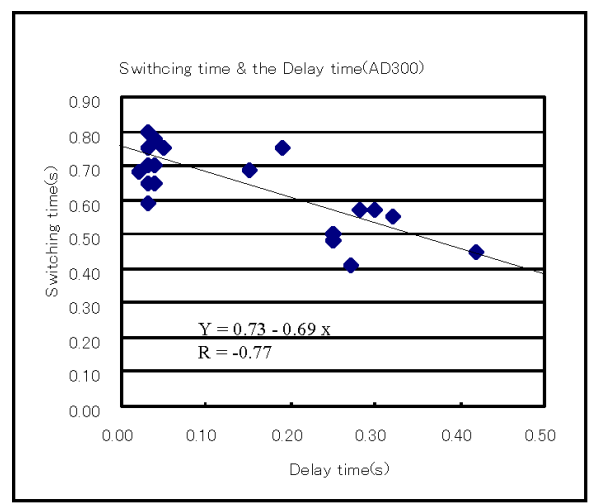

Fig. 7. A negative correlation between switching time and the delay time.

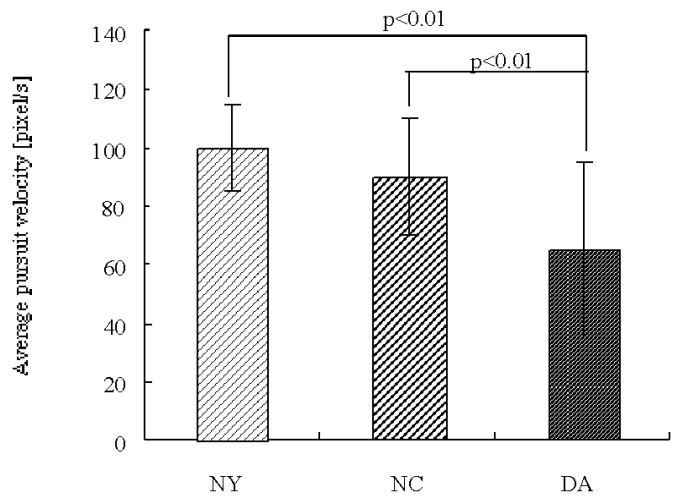

Fig. 8. Comparison of averaged tracking velocity (= pursuit velocity) 


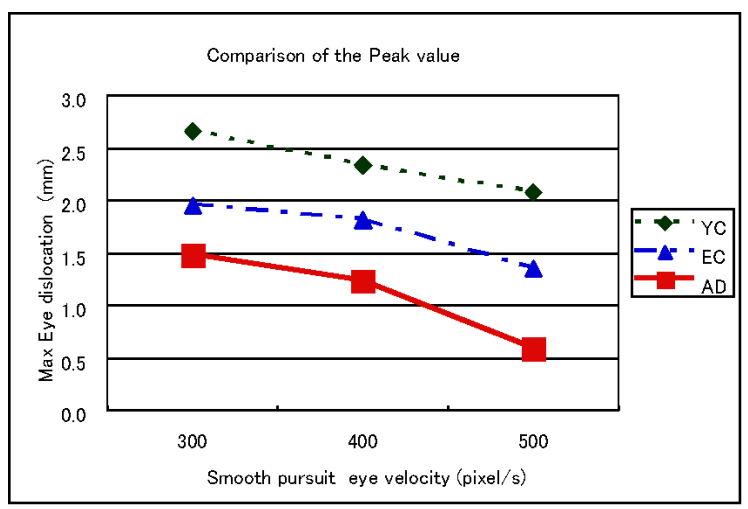

Fig. 9. Peak values of the subjects.

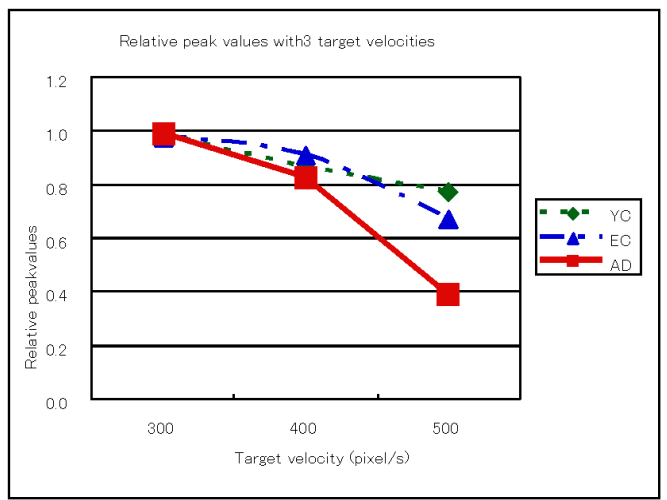

Fig. 10. The relative peak values.

2. The peak values of the eye locations have the same order as the velocities, namely $A D<E C<Y C$. The gpeak valueh here means the maximum eye movement at the switching time point of internal and external rectus muscles. The peak values decrease naturally because the distances for eye to meet the target become shorter in proportion to the target velocities. But the rates of decreasing are not same in the three types of subjects but they are clearly different and line up in the order, namely $A D>E C>Y C$. (Fig.9)

3. The relative peak value drastically falls down especially in AD patients with target velocity of 500 pixels/s. (Fig.10) It suggest that the eye controlling mechanism in AD patient can not catch up the speed of the target probably because of brain degeneration.

4. A correlation coefficient between the target movement and the eye movement is also smallest in the AD patient with target velocity of 300 pixels/s. (Fig.11) It is interesting this time that the difference between AD and other 


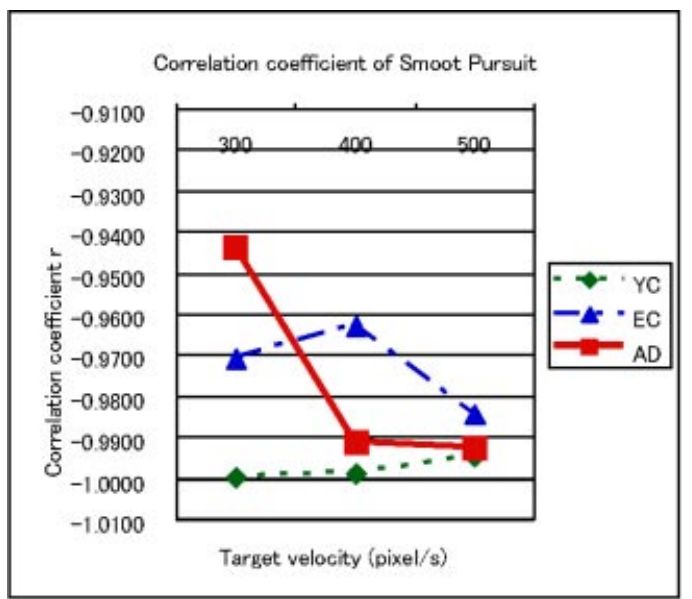

Fig. 11. Correlation coefficient of Smoot Pursuit.

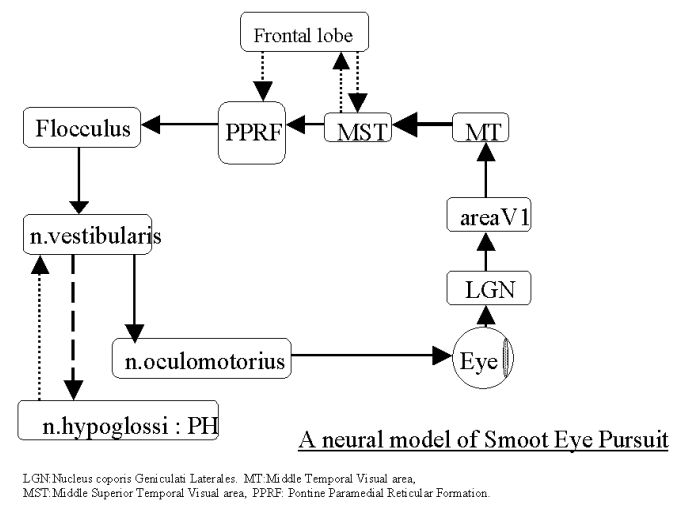

Fig. 12. A neural model of smooth pursuit eye movement.

subject groups becomes most clear in the slowest target velocity. The phenomena may be caused by the demented patients' difficulty to concentrate his attention during slow pursuing problem.

\section{A Neural Model of the Smooth Pursuit}

From a viewpoint of brain anatomy and neuron physiology, the mechanism of smooth pursuit eye movement is not clear yet. [4] As many study on several animal experiments have given us some standpoints as is shown in Fig.12. This figure indicates that the most part of the neural network of smooth pursuit are included in the just deteriorated part of the demented brain such as frontal lobe and parietal lobe. Considering the participation of widely spread neural network 
for the smooth pursuit, it seems not astonishing that the demented patients show the deviated response in oculogyration including smooth pursuit. (Fig.12)

\section{Conclusions}

Human oculogyration may become a new diagnosing method for the Alzheimer type dementia because the smooth pursuit of the demented patients has shown clear deviations from the healthy elders. Especially the pursuit velocity, peak value, switching time and correlation coefficient seems to be useful to discriminate the demented patients from the healthy subjects.

\section{Acknowledgment}

We extend sincere thanks to Dr. Shogo Fukushima in Matsushita Denko who has developed the measuring system and Dr. Xue Ming Shi who has executed the clinical experiments supervising other postgraduate students in our institute. We would like to thank also many patients and volunteers who have kindly participated in the study.

\section{References}

1. A.Jones, R.P.Friedland, B.Koss, L.Stark and B.A.Thompkins-Ober: Saccadic intrusions in Alzheimer-type dementia, J.Neurol, Vol.229, pp189-194, 1983.

2. Hutton JT, Nagel JA, Loewenson RB: Eye tracking dysfunction in Alzheimer-type dementia, Neurology vol.34,pp99-102,1984.

3. Robinson, D.A., Gordon, J. L. and Gordon, S. E.: A model of the smooth pursuit eye movement system,Biological Cybernetics, vol.55, pp43-57. 1988.

4. Lisberger, S.G., Morris, E.J. and Tychsen. L.: Visual motion processing and sensorymotor integration for smooth pursuit eye movements. Annual Review Neuro-science, vol.10, pp97-129. 1987.

5. G.Zaccara, P.F.Gangemi, G.C.Muscas, M.Paganini, S.Pallanti, A.Parigi, A.Messori and G.Arnetoli: Smooth-pursuit eye movements: alterations in Alzheimer's disease, J.Neurological Sciences, Vol.112, pp81-89, 1992

6. Ichiro FUKUMOTO:A computer simulation of the new diagnosing method by human eye light-reflexes,Proceedings of IEEE-EMBS Asia-Pacific Conference on Biomedical Engineering( APBME2000), pp624-625, 2000.9.26

7. Ichiro FUKUMOTO:A basic study for the new dementia diagnostic system using human eye light reflex, Proceeding, International Workshop GerontechnologyAge-Related Change in Human Factors and Application Technology, 2001.3.13Tue.16 Fri.

8. Ichiro Fukumoto: A basic study of new dementia diagnosing methods by neuro physiological measurement and computer aided parameter visualizing technique, IEICE TRANS.FUNDAMENTALS, Vol.E82-, No.1 2001.6 